\title{
La metamorfosis del liderazgo educativo. Hacia nuevas tendencias
}

\author{
MARÍA AMPARO CALATAYUD-SALOM ${ }^{a}$
}

pp. 7-12

RESUMEN El presente artículo evidencia, en líneas muy generales, los cambios acontecidos en la evolución del concepto de liderazgo, a la luz de las nuevas exigencias y retos con los que se enfrenta en la actualidad el liderazgo educativo. Ante las nuevas exigencias de la sociedad de la información y de la incertidumbre, de las investigaciones nacionales e internacionales, emergen nuevas tendencias y visiones del liderazgo que nos sitúan ante su metamorfosis, vertebrada por las bases de un modelo transformacional, distribuido, compartido, participativo y resonante. Es decir, de un liderazgo pedagógico que ha de convertir al director en un profesional reflexivo que analiza, de manera colaborativa, las prácticas directivas vinculadas a los contextos sociales y políticos de referencia. Un liderazgo que posibilite esclarecer los entresijos y mejorar aquellos aspectos del centro que interfieran de forma negativa en el camino hacia la calidad y hacia la mejora de los aprendizajes de los estudiantes.

PALABRAS CLAVE dirección, liderazgo pedagógico, mejora, organización escolar.

\section{HISTORIA DEL ARTÍCULO}

¿CÓMO CITAR?:

Calatayud-Salom, M.A. (2017). La metamorfosis del liderazgo educativo. Hacia nuevas tendencias. Perspectiva Empresarial, 4(2), 7-12. http://dx.doi.org/10.16967/rpe. v4n2a1

RECIBIDO: 28 de abril de 2017 APROBADO: 20 de agosto de 2017

CORRESPONDENCIA:

María Amparo Calatayud-Salom, Departamento de Didáctica y Organización Escolar, Facultad de Filosofía y Ciencias de la Educación, Universidad de Valencia, Avda. Blasco Ibáñez, 30. C. P. 46010, Valencia, España. 


\section{¿CóMO CITO EL ARTÍ́CULO? HOW TO CITE THIS PAPER?}

\section{CHICAGO:}

Calatayud-Salom, María Amparo. 2017. "La metamorfosis del liderazgo educativo. Hacia nuevas tendencias". Perspectiva Empresarial 4(2): 7-12. http:// dx.doi.org/10.16967/rpe. v4n2a1

MLA:

Calatayud-Salom, María Amparo. "La metamorfosis del liderazgo educativo. Hacia nuevas tendencias". Perspectiva Empresarial 4.2 (2017): 7-12. Digital. http:// dx.doi.org/10.16967/rpe. v4n2a1

\section{The metamorphosis of educational leadership. Towards new trends}

ABSTRACT This article shows, grosso modo, the changes that have taken place in the evolution of the concept of leadership, in light of the new demands and challenges faced by educational leadership today. As regards the new demands of the information and uncertainty society, and of national and international research, new trends and views of leadership emerge that place us before its metamorphosis, held up by the bases of a transformational, distributed, shared, participatory, resonant model. In other words, pedagogical leadership must turn the principal into a sensible professional that collaboratively analyzes the management practices linked to the social and political contexts of reference, while being able to clarify the ins and outs and improve those central aspects that interfere negatively with the quality and improvement of students' learning.

KEYWORDS Keywords: management, pedagogical leadership, improvement, school organization.

\section{A metamorfose da liderança educativa. Em direção a novas tendências.}

RESUMO 0 presente artigo evidencia, em linhas muito generalizadas, as mudanças ocorridas na evolução do conceito de liderança, a partir das novas exigências e desafios com os quais a liderança educativa se confronta na atualidade. Diante das novas exigências da sociedade da informação e da incerteza, das pesquisas nacionais e internacionais, emergem novas tendências e visões de liderança que nos posicionam frente à sua metamorfose, vertebrada pelas bases de um modelo transformacional, distribuído, compartilhado, participativo e ressonante, aliás, de uma liderança pedagógica que converterá ao diretor em um profissional reflexivo que analisa, de maneira colaborativa, as práticas diretivas vinculadas aos contextos sociais e políticos de referência. Uma liderança que possibilite solucionar os emaranhados e melhorar os aspectos do centro educacional que interfiram de forma negativa no caminho em direção à qualidade e melhora da aprendizagem dos estudantes.

PALAVRAS CHAVE direção, liderança pedagógica, melhora, organização escolar. 


\section{Introducción}

El liderazgo, al igual que otros tantos conceptos organizativos, ha estado influenciado por el mundo de la ciencia empresarial (que es de dónde surgen) y la psicología social. El estudio de este fenómeno ha evolucionado a lo largo del tiempo, de modo que generó bastante literatura durante todo el siglo anterior, y lo continúa haciendo hasta el momento (Contreras, 2016; Freire \& Miranda, 2014; Martínez, 2014; Murillo, 2017; Pérez, 2008). Esta evolución ha pasado por una serie de etapas que es posible identificar claramente. Desde la teoría de rasgos, hasta la consideración existente, en la actualidad, entre el comportamiento del líder y la cultura organizativa. Del mismo modo, los distintos paradigmas organizativos han definido, de forma diferente, el discurso del liderazgo. A continuación, se presenta cada uno de estos enfoques.

TABLA 1. Evolución del liderazgo según los paradigmas

\begin{tabular}{ll}
\hline PARADIGMAS & LIDERAZGO \\
\hline CIENTÍFICO & $\begin{array}{l}\text { Liderazgo formal, autoridad definida } \\
\text { por conocimientos técnicos y } \\
\text { funciones pragmáticas y burocráticas. }\end{array}$ \\
\hline \multirow{2}{*}{ INTERPRETATIVO } & $\begin{array}{l}\text { Liderazgo personal, autoridad } \\
\text { difusa y cambiante, centrada en la } \\
\text { aceptación personal y las funciones, } \\
\text { básicamente, de animación de } \\
\text { grupos. }\end{array}$ \\
\hline SOCIO-CRITICO & $\begin{array}{l}\text { Liderazgo ideológico, la autoridad } \\
\text { centrada en la fuerza política y } \\
\text { las funciones de distribución de } \\
\text { poder institucional y resolución de } \\
\text { conflictos. }\end{array}$ \\
\hline $\begin{array}{l}\text { Liderazgo participativo, } \\
\text { transformacional y resonante. } \\
\text { Autoridad centrada en la colaboración } \\
\text { e innovación que logrará conducir } \\
\text { a la comunidad educativa, tanto } \\
\text { hacia la consecución del proyecto } \\
\text { institucional compartido, como } \\
\text { hacia el cambio y la mejora de la } \\
\text { organización. }\end{array}$ \\
\hline
\end{tabular}

Fuente: elaboración propia.

Como se ha observado, cada uno de estos paradigmas comporta una visión distinta de entender el liderazgo. Como señala Yáñez (2003, p. 303):

No es de extrañar que el constructo de liderazgo dominante en el momento actual es, desde la década de los 90, el de liderazgo transformacional. Por liderazgo transformacional entendemos que es aquel líder que se ocupa de la transformación de la dimensión cultural de la organización estimulando la independencia como base de la acción y el cambio.

Hoy por hoy, los debates actuales sobre liderazgo en educación están presididos por los modelos transformacionales, que se presentan como una imagen capacitadora que mantiene vínculos positivos tanto con la cultura de la institución educativa como con el rendimiento académico de los alumnos.

Concretamente, es a partir de la teoría de la contingencia cuando verdaderamente se puede hablar de nuevas perspectivas, las cuales comenzaron con los trabajos de autores como Burns y Bass. A partir de esto, empieza a hablarse de liderazgo transaccional y de liderazgo transformacional. En España se encuentran estudios interesantes realizados sobre estos dos tipos de liderazgo (Bolívar, 2010; Murillo, 2006; 2017).

Es en la década de los noventa cuando, por primera vez, comienza a relacionarse el liderazgo con la concepción de poder y de micropolítica planteada por Ball (1989), y se enfoca más la atención sobre aspectos culturales que sobre los estructurales de la organización (transformar la cultura del centro va a ser uno de los objetivos básicos). Por lo tanto, esta nueva idea que se gesta sobre el liderazgo se caracteriza, básicamente, por aspectos resumidos con claridad por Yáñez \& Sánchez (1994):

- El liderazgo comienza a contemplarse más como un proceso a través del cual se gestionan y construyen significados que comparten los miembros de una organización. Se enfatiza, por tanto, la dimensión simbólica y cultural de los procesos de liderazgo.

- La idea de visión propia del modelo transformacional o carismático, visionario, etc., se entiende como una imagen del futuro que articula los valores, los propósitos y la identidad de los miembros de la organización, o como el conjunto de creencias sobre cómo tales miembros deberían actuar e interactuar para alcanzar algún estado futuro idealizado.

- La función del liderazgo consiste en el mantenimiento y, a la vez, la modificación de la cultura. 
- La idea según la cual el liderazgo está condicionando de una manera sustancial por la forma en que se conciben las relaciones de poder en las escuelas y las fuentes de legitimidad de la autoridad en ellas. Es decir, el liderazgo se define en términos de las relaciones de poder entre los miembros de una organización.

- El liderazgo se percibe como un proceso dirigido a facilitar el cambio.

La dicotomía planteada entre el liderazgo transaccional y el transformacional supone un avance importante y un cambio radical en la forma de entender y estudiar el liderazgo. Hunt (1999) argumenta que es a partir de este momento cuando se puede hablar, realmente, del surgimiento de un nuevo paradigma del liderazgo.

Por liderazgo transaccional se entiende el intercambio entre líder y seguidores, de forma que este es quien proporciona recompensas para que los seguidores se conformen a sus deseos. En cambio, el liderazgo transformacional, también denominado "liderazgo carismático", "visionario", etc., se ocupa de la transformación de la dimensión cultural de la organización al estimular la independencia como base de la acción y el cambio.

Como se puede intuir, en estos momentos se aboga por un liderazgo que presente una función estratégica compartida y comprometida en su dimensión transformacional, así como en su papel potenciador de la cultura. En la tabla 2 se sintetizan las principales ideas asociadas a cada uno de estos estilos de liderazgo:

Como se observa en la tabla 2, el líder transformacional será quien ayudará a potenciar en el centro un clima de trabajo que favorezca el compromiso de los docentes en un proyecto elaborado en común y, por tanto, compartido. Su trabajo contribuirá a mejorar la capacidad de todos los miembros de la organización para resolver todos los problemas que se le planteen. A este respecto, es relevante destacar uno de los estudios realizados por Leithwood \& Jantzi (1990), en el cual, entre sus resultados, se concluye que los directores que desarrollan estrategias transformacionales promueven en sus centros culturas colaborativas. Concretamente, las estrategias que utilizaban los directores de la muestra para influir en la cultura de la escuela eran: 1 . Reforzar la cultura de la escuela: (implica dar prioridad a determinados objetivos compartidos, etc.); 2 . Realizar una buena gestión (organizar mejor el tiempo, conseguir mejores recursos económicos, etc.); 3. Impulsar el desarrollo de los profesores; 4. Establecer una comunicación directa y frecuente; 5. Compartir el poder y la responsabilidad con otros; y 6 . Utilizar símbolos y rituales para expresar los valores culturales (la exteriorización de símbolos propios hace más visibles los contenidos relevantes de la cultura).

Como se puede intuir, la tendencia actual en el campo de la organización escolar es la de identificar al líder transformacional como el liderazgo más importante a desarrollar en las instituciones escolares. Además, como ya se ha comentado con anterioridad, es el liderazgo vigente en las organizaciones que aprenden, etc. A este liderazgo, junto con la importancia de las estructuras y la cultura escolar, se les identifica como las variables más determinantes en los procesos de aprendizaje organizativo, tanto en los centros escolares, como en las organizaciones no educativas. Además, es el liderazgo que se propone como modelo en la reestructuración escolar, a fin de que las escuelas puedan hacer frente a los desafíos actuales, nacidos

TABLA 2. Diferencias entre liderazgo transaccional vs. transformacional

\begin{tabular}{|c|c|}
\hline LIDERAZGO TRANSACCIONAL & LIDERAZGO TRANSFORMACIONAL \\
\hline $\begin{array}{l}\text { - Basado en intercambio líder-seguidores para alcanzar } \\
\text { metas de grupo: esfuerzo y lealtad a cambio de } \\
\text { recompensas tangibles. } \\
\text { - Los seguidores también pueden afectar al líder, quien } \\
\text { debe ajustar su estilo según el comportamiento de } \\
\text { aquellos. } \\
\text { - Sistemas centralizados y estáticos. } \\
\text { - Prioridad en el mantenimiento. } \\
\text { - Cambios organizativos claramente delimitados que no } \\
\text { implican cambios en estructuras y/o relaciones. }\end{array}$ & $\begin{array}{l}\text { - Basado en implicación de los participantes en mejorar la } \\
\text { organización con el compromiso de todos. } \\
\text { - El líder cambia valores, creencias y necesidades de los } \\
\text { seguidores a partir de una idea de misión organizativa. } \\
\text { - Sistemas descentralizados y dinámicos. } \\
\text { - Prioridad en el desarrollo. } \\
\text { - Cambios complejos y dinámicos orientados a transformar } \\
\text { la cultura y el diseño de la organización. }\end{array}$ \\
\hline
\end{tabular}


de la sociedad presente, caracterizada por la incertidumbre, la complejidad y la ambigüedad.

\section{Método}

El presente artículo se fundamenta en una metodología puramente descriptiva, ya que pretende dar una visión general sobre las nuevas tendencias del liderazgo en educación. La literatura ha señalado la importancia del liderazgo en la mejora de las organizaciones y en el desarrollo de la innovación (Bolívar, 2010; Contreras, 2016; Freire y Miranda, 2014; Martínez, 2014; Murillo, 2006; Murillo, 2017; Pérez, 2008). Es por ello que en este artículo se analiza la información con base en el propósito de presentar los tipos de liderazgo emergentes en la actualidad. Estos liderazgos surgen con el fin de dar respuesta a las demandas y necesidades de la sociedad actual.

\section{Nuevas tendencias del liderazgo en las organizaciones educativas}

En la actualidad, y al tomar como referente el modelo transformacional, han emergido en las últimas décadas nuevas ideas en relación con el liderazgo que son dignas de consideración. Por ejemplo, entre las más relevantes se destacan las aportaciones siguientes:

- Conley y Goldman (1994) desarrollan la idea del líder educativo como gestor de la energía flotante dentro de la escuela y utilizan el término de liderazgo facilitador. Según estos autores, el liderazgo facilitador "incluye comportamientos que ayudan a la organización a cumplir metas que deben ser compartidas, negociadas o complementadas" (p. 238).

- Bryman (1996) trata el liderazgo disperso o distribuido que, como su nombre indica, se encuentra disperso entre los diferentes miembros del equipo, del centro. Concretamente, se define como la capacidad que puede atribuirse no solo a un individuo, sino también a un conjunto de individuos, de unidades organizativas, etc.

- Goleman et al. (2013) presentan el liderazgo resonante, un tipo de liderazgo que sintoniza con los sentimientos de las personas y los encauza en una dirección emocionalmente positiva.

- El liderazgo clarividente, propuesto por Sharma, que intenta vislumbrar la visión de futuro de la organización.

- El liderazgo sin límites planteado por Heifrtz y Linsky, el cual entiende el liderazgo como una figura a la que no le importa que surja el conflicto e intenta ver las cosas de otra manera.

- El liderazgo para la innovación propuesto por Villa, en el que se incide en la relevancia de la innovación y la mejora continua.

- Calatayud (2016) señala el liderazgo interesado, aquel que busca solo el éxito personal frente al organizacional. Toma las decisiones en busca siempre del éxito propio, pero hace ver a sus seguidores que el éxito es para el centro educativo, en su conjunto.

\section{Conclusiones}

De todo lo comentado anteriormente se deduce una idea clara, y es que en la actualidad existe la necesidad de un líder comprometido con el cambio, con la búsqueda de nuevas formas de organización, directores que inciten y apoyen la transformación de la enseñanza y de la escuela, de equipos directivos que sean vertebradores de la dinámica colegiada del centro, capaces de propiciar el trabajo en equipo de los docentes y el ejercicio de la autonomía pedagógica, organizativa y de gestión de los centros (Calatayud, 2008; 2008a; 2009; 2013; 2015; 2016). Para ello, se necesita de un líder que reúna básicamente estas competencias: 1 . Una persona con capacidad de orientar el cambio en su organización, que sepa comunicar y tenga la habilidad de conseguir los objetivos marcados con el apoyo de toda la comunidad; 2 . Que ejerza una influencia sobre las personas que componen una comunidad educativa, sin utilizar el poder o autoridad formal; 3. Con capacidad de mediar entre los componentes de la organización y orientar en el aprendizaje; 4. Influyente en la comunidad y que guíe positivamente la actividad del resto hacia los objetivos de la organización; 5. Con un conocimiento amplio de la institución, y tenga claro y presente el objetivo de la organización; 6 . Que conciba el conflicto como algo positivo para la organización; 7. Con capacidad de motivar, 
comunicar y potenciar al resto de los miembros de la organización; 8. Sabe implicarse, ser transparente, saber confiar y delegar responsabilidades, así como crear buenas relaciones; 9. Sea el motor de la organización, y tenga mente abierta y visión de futuro; y 10. Tenga compromiso ético y responsabilidad.

Estas competencias que se fundamentan en el ejercicio de un liderazgo responsable y comprometido con la educación, y al que le corresponde velar porque la institución educativa sea capaz de proporcionar aprendizajes valiosos y de calidad para todos los estudiantes (Bolívar, 2010; Contreras, 2016; Freire \& Miranda, 2014; Martínez, 2014; Murillo, 2017; Pérez, 2008). En definitiva, un liderazgo asentado en las bases de un modelo transformacional, distribuido, compartido, participativo y resonante. Es decir, de un liderazgo pedagógico que ha de lograr que el director se convierta en un profesional reflexivo, quien analiza de manera colaborativa las prácticas directivas vinculadas a los contextos sociales y políticos de referencia. En fin, un liderazgo que posibilite esclarecer los entresijos y busque mejorar aquellos aspectos del centro que interfieran de forma negativa en el camino hacia la calidad y hacia la mejora de los aprendizajes de los estudiantes. Es necesario, por tanto, una metamorfosis del liderazgo acorde con las nuevas exigencias del contexto, lo cual facilite dar respuesta a los desafíos de la profesión directiva en la actualidad.

\section{REFERENCIAS}

Contreras, T. (2016). Liderazgo pedagógico, liderazgo docente y su papel en la mejora de la escuela: una aproximación teórica. Propósitos y representaciones, 4(2), 231-284.

Bolívar, A. (2010) ¿Cómo un liderazgo pedagógico y distribuido mejora los logros académicos? Revisión de la investigación y propuesta. Magis, 3(5), 85-106.

Ball, S. (1989). La micropolítica de la escuela. Hacia una teoría de la organización escolar. Madrid: Paidós.

Bryman, A. (1996). Leadership in organizations. En N. Glegg (Coord), Handbook of Organization Studies, (pp. 52-67). Thousand Oaks: SAGE.

Calatayud-Salom, A. (2008). La escuela del futuro. Hacia nuevos escenarios. Madrid: CCS.

Calatayud-Salom, A. (2008a). El liderazgo educativo en un mundo globalizado: hacia nuevas visiones. En J. Gairin, \& S. Antúnez, (Ed.), Organizaciones educativas al servicio de la sociedad. Madrid: Wolters Kluwer.

Calatayud-Salom, A. (2009). Entresijos de los centros escolares. Desvelarlos para mejorarlos. Málaga: Aljibe.

Calatayud-Salom, A. (2013). El liderazgo emergente que reclaman hoy los docentes en las organizaciones escolares. En VI Congreso Internacional sobre Dirección de centros Educativos C.D. Facultad de Psicología y Educación de la Universidad de Deusto.

Calatayud-Salom, A. (2015). El liderazgo emergente de los directores escolares en España. La voz del profesorado. Revista Iberoamericana de Educación (OEI), 69.

Calatayud-Salom, A. (2016). Necesidad y exigencia de un cambio cultural en la dirección escolar. Educaweb.com, 340.

Conley, D., \&, y Golman, P. (1994). Ten propositions for facilitative leadership. En J. Murphy, \& K. S. Louis (Ed), Reshapihg the Principalship: Insights from transformational reform efforts. Corwin Press-California.

Freire, S, \& Miranda, A. (2014). El rol del director en la escuela: el liderazgo pedagógico y su incidencia sobre el rendimiento académico. Lima: GRADE. Avances de Investigación, 17.

Goleman, D. et al. (2013). Liderazgo. El poder de la inteligencia emocional. Barcelona: Ediciones B.

Hunt, J. (1999). Transformational leadership's transformation of the field. An historical essay. Leadership Quarterly, 10, 129-144.

Leithwood, K. (1994). Liderazgo para la reestructuración de las escuelas. Revista de Educación, 304, 31-60.

Leithwood, K., \& Jantzi, D. (1990). Transformacional leadership. How principals can help reform school cultures. School effectiveness and school improvement, 1(4), 249-280.

Martínez, Y. (2014). El liderazgo transformacional en una institución educativa pública. Educación, 23(44), 7-28.

Murillo, J. (2006). Una dirección escolar para el cambio: del liderazgo transformacional al liderazgo distributivo. Revista electrónica sobre calidad, eficacia y cambio en educación, 4(4e), 11-24.

Murillo, J. (2017). Avances en liderazgo y mejora de la educación. Madrid: RILME.

Pérez, S. (2008). El liderazgo facilitador del aprendizaje organizativo en la escuela (Tesis para optar el grado de Magíster en Educación con mención en Gestión de la Educación, Lima, Pontificia Universidad Católica del Perú, Escuela de Graduados).

Yáñez, J., Sánchez-Moreno, M., \& Murillo, P. (2003). Dirección de centros educativos. Madrid: Síntesis.

Yáñez, J., \& Sánchez, M. (1994). Para comprender las organizaciones escolares. Sevilla: Repiso Libros. 